\title{
ANATOMÍA Y MORFOLOGÍA DE PLANTAS MICROPROPAGADAS-ACLIMATADAS DE Agave potatorum Zucc. FERTIRRIGADAS EN VIVERO
}

\author{
ANATOMY AND MORPHOLOGY OF MICROPROPAGATED-ACCLIMATIZED \\ PLANTS OF Agave potatorum Zucc. FERTIGATED IN NURSERY
}

\author{
Silvia Luna-Luna, J. Raymundo Enríquez-del Valle, Gerardo Rodríguez-Ortiz*, \\ José C. Carrillo-Rodríguez y Vicente A. Velasco-Velasco
}

Instituto Tecnológico del Valle de Oaxaca ITVO. Ex-Hacienda de Nazareno, Santa Cruz Xoxocotlán, Oaxaca, México. 71230. Tel. 01(951) 5170788.

*Autor para correspondencia (grodriguez.itvo@yahoo.com).

\section{RESUMEN}

\begin{abstract}
Agave potatorum Zucc. es una especie silvestre, colectada sin planes de manejo, por lo que se propone propagarla in vitro. El objetivo de la investigación fue evaluar las características morfológicas y anatómicas de plantas de $A$. potatorum micropropagadas-aclimatadas y fertirrigadas con cantidades diferentes de nutrimentos en vivero. Durante los años 2014-2015 se estableció un experimento en vivero, donde 180 plantas micropropagadasaclimatadas se separaron en seis grupos de 30 plantas para fertirrigarlas dos veces por semana durante ocho meses, con una de seis diluciones de la solución universal de Steiner $(5,20,40,60,80$ y $100 \%)$. Transcurridos ocho meses, se evaluó el área foliar (AF), área de la sección transversal de la hoja más grande en su parte basal (AsT), número de haces vasculares (AV), materia seca acumulada en la parte aérea ( $P A=$ tallo + hojas), en la raíz $(R)$ y total $(M S T=P A+R)$. Las plantas crecieron en relación directa con la cantidad de nutrimentos que recibieron. Las plantas fertirrigadas al 5 y al $100 \%$ de concentración de nutrimentos tuvieron respectivamente: 529.4 y $965.6 \mathrm{~cm}^{2}$ de AF, 1.4 y $2.0 \mathrm{~cm}^{2}$ de AsT, 201.5 y 263.5 de AV, 18.9 y $37.3 \mathrm{~g}$ de PA, 8.1 y $12.0 \mathrm{~g}$ de $\mathrm{R}$ y $27 \mathrm{y} 49 \mathrm{~g}$ de MST.
\end{abstract}

Palabras clave: Agave potatorum, haces vasculares, materia seca acumulada, nutrición vegetal.

\section{SUMMARY}

Agave potatorum is a wild species collected without management plans; thus, this research proposes in vitro propagation. This research evaluated morphological and anatomical characteristics of micropropagatedacclimatized $A$. potatorum plants fertigated with different nutrient amounts in the nursery. In 2014 and 2015, a nursery experiment was established and contained 180 micropropagated-acclimatized plants separated into six groups of 30 plants. Each group was fertigated twice a week for eight months with one of six dilutions of Steiner's universal solution $(5,20,40,60,80$ and $100 \%)$. After eight months, leaf area (LA), area of the cross-section of the largest leaf in its basal part (CSA), number of vascular bundles (VB), dry biomass accumulated above ground $(A G=$ stem + leaves), dry biomass in the root $(R)$ and total biomass $(T=A G+R)$ were evaluated. Plants grew in direct relation to the amount of nutrient they received. Plants fertigated at 5 and 100 $\%$ concentration of nutrients, had respectively: 529.4 and $965.6 \mathrm{~cm}^{2}$ of LA, 1.4 and $2.0 \mathrm{~cm}^{2}$ of CSA, 201.5 and 263.5 of $\mathrm{VB} ; 18.9$ and $37.3 \mathrm{~g}$ of AG, 8.1 and $12.0 \mathrm{~g}$ of $\mathrm{R}$, and 27 and $49 \mathrm{~g}$ of $\mathrm{T}$.

Index words: Agave potatorum, vascular bundles, accumulated dry matter, plant nutrition.

\section{INTRODUCCIÓN}

Las especies de Agave son recursos de zonas áridas y semiáridas de México (Pérez Molphe et al., 2012), de las que se obtiene materia prima para la industria de bebidas alcohólicas y de fibras naturales (Martínez-Ramírez et al., 2014). Las poblaciones de varias especies están disminuyendo debido a su aprovechamiento no planificado (SEMARNAT, 2010), por lo que una alternativa para incrementar las poblaciones es propagarlas in vitro y establecer plantaciones. Hay antecedentes de micropropagación de A. fourcroydes Lem. (Garriga et al., 2010), A. tequilana Weber (Angeles-Espino et al., 2012), A. grijalvensis B. Ullrich (Santíz et al., 2012), A. angustifolia Haw. (Monja-Mio et al., 2015), que describen los procedimientos de propagación in vitro, la transferencia de las plantas a sustrato e invernadero para su aclimatización. No obstante, según Pospíšilová et al. (2000), las plantas micropropagadas poseen características morfológicas y fisiológicas que dificultan su transferencia directa a condiciones de campo, por lo que deben someterse a una etapa de aclimatación.

En A. angustifolia se demostró que las plantas micropropagadas responden positivamente al abastecimiento de nutrimentos mediante fertirriego (Enríquez-del Valle et al., 2012), lo que permite establecen en vivero durante ocho a 12 meses, donde crecen al tamaño adecuado para trasplantarlas a campo. No hay suficiente información sobre el abastecimiento nutrimental a plantas micropropagadas de A. potatorum establecidas en vivero, por lo que el objetivo del presente estudio fue evaluar las características morfológicas y anatómicas de plantas de A. potatorum Zucc, micropropagadas-aclimatadas y fertirrigadas en vivero durante ocho meses con cantidades diferentes de nutrimentos. 


\section{MATERIALES Y MÉTODOS}

El presente trabajo se realizó durante los años 2014 y 2015 en el laboratorio de cultivo de tejidos vegetales, invernadero y vivero del Instituto Tecnológico del Valle de Oaxaca, México, localizado a $17^{\circ} 02^{\prime} \mathrm{N}, 96^{\circ} 44^{\prime} \mathrm{O}$ y altitud de $1530 \mathrm{~m}$. Se obtuvieron in vitro 220 plantas de A. potatorum mediante la proliferación de brotes adventicios y su posterior enraizado. Las plantas micropropagadas se transfirieron individualmente a macetas de $300 \mathrm{~cm}^{3}$ con sustrato de perlita-turba de musgo (1:1) y se establecieron durante 70 días en invernadero con 80-90 \% de humedad relativa mediante un sistema de riego por nebulización intermitente de $10 \mathrm{~s}$ cada $12 \mathrm{~min}$, de 10:00 a 15:00 h diariamente. Se utilizó radiación solar difusa, que a medio día era de $400 \mu \mathrm{mol} \mathrm{m} \mathrm{m}^{-2} \mathrm{~s}^{-1}$.

Transcurridos $70 \mathrm{~d}$ las plantas se establecieron durante $150 \mathrm{~d}$ en vivero, expuestas a menor humedad relativa, radiación solar disminuida $40 \%$ mediante malla sombra, que

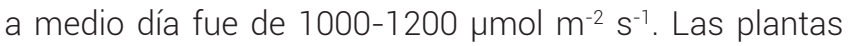
se fertirrigaron a nivel de sustrato dos veces por semana con solución nutritiva (SN) de la formulación universal de Steiner (Steiner, 1984) diluida a $20 \%$. Todo lo anterior de acuerdo con el procedimiento descrito por Enríquez et al. (2016). Transcurridos los 150 d en vivero se eligieron 180 plantas homogéneas en tamaño y de apariencia sana y se trasplantaron en contenedores individuales de polietileno de $1529 \mathrm{~cm}^{3}$ con tierra agrícola, que se analizó conforme a la norma oficial mexicana NOM-021-RECNAT-2000 (SEMARNAT, 2002).

Las características de la tierra fueron: textura francoarenosa (método del hidrómetro de Boyoucous), macrominerales: $1.21 \mathrm{mEq}$ de $\mathrm{K} 100 \mathrm{~g}^{-1}$ y $87.75 \mathrm{mg}$ de $\mathrm{P} \mathrm{kg}^{-1}$; $\mathrm{pH} 7.7$ (método del potenciómetro), conductividad eléctrica 0.46 dS $\mathrm{m}^{-1}$ y materia orgánica $1.92 \%$. El total de plantas se separó en seis grupos de 30, y cada grupo se fertirrigó con la SN Steiner, a una de las diluciones siguientes: 5, 20, 40,60, 80 y $100 \%$ de concentración de nutrimentos y pH ajustado a 5.8. La cantidad de nutrimentos en $\mathrm{mg} \mathrm{L}^{-1}$ de la SN al 100 \% fue: 102.6 N, 30.6 P, 220.1 K, 182.3 Ca, 49.0 Mg y 110.9 S. Cada planta se fertirrigó con $200 \mathrm{~mL}$ de SN dos veces por semana durante ocho meses. El experimento se estableció de acuerdo con un diseño completamente al azar, usando una planta como unidad experimental con 30 repeticiones por tratamiento.

Al inicio del experimento se tomaron al azar 12 plantas para cuantificar área foliar $\left(\mathrm{cm}^{2}\right)$ mediante escaneo y uso de software de procesamiento de imágenes (ImageJ®), volumen foliar $\left(\mathrm{cm}^{3}\right)$ mediante desplazamiento de agua en una probeta graduada y la suculencia, que entre diversos criterios para describirla (Mantovani, 1999) se eligió el mé- todo de Salisbury y Ross (1992) como la relación volumen foliar/área foliar $\left(\mathrm{cm}^{3} \mathrm{~cm}^{-2}\right)$. En la parte basal de la hoja más grande se realizó un corte transversal de $0.5 \mathrm{~mm}$ de espesor, que se colocó sobre un portaobjetos con una escala milimétrica, en el que se observaron al microscopio (40x) y contabilizaron los haces vasculares y se determinó el área de la sección transversal mediante ImageJ $\AA^{\circledR}$. Las muestras de la parte aérea (tallo + hojas) y de la raíz se secaron en estufa de convección a $70{ }^{\circ} \mathrm{C}$ hasta peso constante y su biomasa se cuantificó en balanza analítica con precisión de $0.1 \mathrm{mg}$. Se obtuvo la relación de biomasa entre la parte aérea y la raíz $(\mathrm{Pa} / \mathrm{R})$; al final del experimento se tomaron al azar ocho individuos por tratamiento para cuantificar las mismas variables de inicio. Los datos fueron analizados en el programa SAS, Versión 9 (SAS Institute, 2002), a través del cual se obtuvo un análisis de varianza y comparaciones de medias (Tukey, 0.05).

\section{RESULTADOS Y DISCUSIÓN}

Las plantas micropropagadas-aclimatadas en invernadero y cultivadas en vivero deben mostrar vigor de crecimiento y morfología apropiada para su establecimiento en campo (Monja-Mio et al., 2015). Durante las etapas de invernadero y vivero, el suministro de nutrimentos fue un factor que promovió que plantas micropropagadas de Agave angustifolia (Enríquez-del Valle et al., 2012), A. americana (Enríquez et al., 2013) y A. potatorum (Enríquez et al., 2016) alcanzaran tamaño mayor. En este experimento, cuando las plantas de A. potatorum se trasplantaron a macetas con tierra agrícola tenían 220 d de crecimiento ex vitro, $120.2 \mathrm{~cm}^{2}$ de área foliar, $22.3 \mathrm{~cm}^{3}$ de volumen foliar y una suculencia de $0.18 \mathrm{~cm}^{3} \mathrm{~cm}^{-2}$.

La sección transversal de la hoja tenía $0.2 \mathrm{~cm}^{2}$ de área, donde se contabilizaron 86.5 haces vasculares. Cuando transcurrieron los $240 \mathrm{~d}$ del experimento, todas las plantas en las diversas condiciones fueron más grandes, en relación positiva con el abastecimiento de nutrimentos. Los análisis de varianza mostraron que las dosis de fertirriego tuvieron efecto diferente altamente significativo $(P \leq 0.01)$ en área foliar, volumen foliar, número de haces vasculares de la sección transversal, área de la sección trasversal de la hoja, materia seca acumulada en la parte aérea, raíz y en la total (Cuadro 1).

Estos resultados difieren de los datos obtenidos por Martínez et al. (2012) y Martínez-Ramírez et al. (2014), quienes evaluaron el crecimiento de plantas de A. potatorum establecidas en campo como monocultivo o asociadas con maíz (Zea mays) o frijol (Phaseolus vulgaris) y que se fertilizaron con regímenes diferentes de nitrógeno $(0,50$, 100 y $150 \mathrm{~kg} \mathrm{ha}^{-1}$ ). Sus resultados mostraron que las dosis de fertilización no tuvieron efectos diferentes en diámetro, 
Cuadro 1. Cuadrados medios y significancia del análisis de varianza de características de plantas de Agave potatorum, con aplicación de diferentes dosis de nutrimentos en vivero.

\begin{tabular}{|c|c|c|c|c|c|c|c|c|c|}
\hline \multirow{3}{*}{ FV } & \multirow{3}{*}{ GL } & \multicolumn{8}{|c|}{ Cuadrados medios y significancia } \\
\hline & & \multirow{2}{*}{ AF } & \multirow{2}{*}{ VF } & \multirow{2}{*}{$\mathrm{HV}$} & \multirow{2}{*}{ AST } & \multicolumn{3}{|c|}{ MS } & \multirow{2}{*}{$\mathrm{Pa} / \mathrm{R}$} \\
\hline & & & & & & T & PA & $\mathrm{R}$ & \\
\hline CS & 5 & $210,034.5 *$ & $45,585.8 * *$ & $20,549.7 *$ & $1.7 *$ & $714.5^{\star \star}$ & $405.8 * \star$ & $37.3 * \star$ & $0.5 \mathrm{~ns}$ \\
\hline$E$ & 42 & $11,895.5$ & 1631.2 & 682.7 & 0.09 & 45.8 & 35.1 & 3.3 & 0.3 \\
\hline To & 47 & & & & & & & & \\
\hline
\end{tabular}

FV: fuente de variación, GL: grados de libertad, CS: diluciones de la solución nutritiva, E: error experimental, To: total, AF: área foliar, VF: volumen foliar, HV: número de haces vasculares de la parte basal de la hoja, AST: área de la sección transversal la parte basal de la hoja, MS: materia seca, T: MS total, PA: MS aérea, R: MS en raíz, Pa/R: relación parte aérea/raíz. * ** valores significativos a P $\leq 0.05$ y P $\leq 0.05$, respectivamente, ns: valores sin efectos significativos $(P>0.05)$.

altura, área foliar y hojas desplegadas de las plantas.

En varias especies vegetales las raíces pueden constituir entre el 20 y el $50 \%$ del peso seco total, y las plantas sometidas a déficit de agua o abastecimiento de nutrimentos acumulan menos biomasa y la mayor proporción de esta biomasa se ubica en las raíces (Martínez et al., 2012), en comparación con plantas que crecen en condiciones óptimas de agua y nutrimentos. En el presente estudio, las plantas de A. potatorum micropropagadas establecidas en vivero y sometidas a dosis diferentes de fertirriego mostraron que del 70.1 al $75.7 \%$ de su materia seca total se acumuló en la parte aérea (tallo + hojas) y del 24.3 al 29.9 \% se acumuló en la raíz (Cuadro 2).

Las plantas abastecidas con diferentes cantidades de nutrimentos no mostraron diferencias significativas en la relación de peso de la parte aérea/raíz. Al inicio del experimento, las hojas de las plantas micropropagadas tenían $0.18 \mathrm{~cm}^{3} \mathrm{~cm}^{-2}$ de suculencia y $240 \mathrm{~d}$ después las hojas de todas las plantas que se abastecieron con las diferentes dosis de nutrimentos habían aumentado en suculencia en- tre 0.30 y $0.41 \mathrm{~cm}^{3} \mathrm{~cm}^{-2}$, que no fueron significativamente diferentes (Cuadro 2).

La suculencia y grosor de las hojas son por causa de la abundancia de células con vacuolas grandes para almacenar agua y acumular ácidos orgánicos durante la noche, característica de las plantas de metabolismo ácido crasuláceo adaptadas a las zonas áridas (Barrera et al., 2014). En el presente estudio, conforme se suministró a las plantas cantidades crecientes de nutrimentos, éstas desarrollaron área foliar y área de la sección transversal basal más grandes, ya que las plantas fertirrigadas a 5 y a $100 \%$ de concentración de nutrimentos tuvieron 529.4 y $965.6 \mathrm{~cm}^{2}$ de área foliar, 1.37 y $2.0 \mathrm{~cm}^{2}$ de área de la sección transversal de la base de la hoja, donde se contabilizaron 201 y 263 haces vasculares, respectivamente, que en cada caso fueron significativamente diferentes $(P$ $\leq 0.01)$.

La capacidad de fotosíntesis de las plantas está relacionada con su área foliar, y en cultivos de olivo (Olea europaea L.) se ha determinado que la condición nutrimental de la

Cuadro 2. Características de plantas de Agave potatorum micropropagadas-aclimatadas y fertirrigadas durante ocho meses en vivero con dosis diferentes de nutrimentos.

\begin{tabular}{|c|c|c|c|c|c|c|}
\hline \multirow{2}{*}{ Var } & \multicolumn{6}{|c|}{ Diluciones (\%) de la solución nutritiva Steiner } \\
\hline & 5 & 20 & 40 & 60 & 80 & 100 \\
\hline MST & $27.0 \pm 3.4 \mathrm{~d}$ & $30.9 \pm 4.5 d$ & $39.1 \pm 4.1 \mathrm{bc}$ & $46.0 \pm 3.5 \mathrm{ab}$ & $48.4 \pm 7.9 \mathrm{ab}$ & $49.3 \pm 12.3 \mathrm{a}$ \\
\hline PA & $18.9 \pm 2.8 c$ & $22.1 \pm 2.8 \mathrm{cb}$ & $29.1 \pm 3.1 \mathrm{ab}$ & $33.3 \pm 2.5 \mathrm{a}$ & $35.2 \pm 5.8 \mathrm{a}$ & $37.3 \pm 9.3 a$ \\
\hline $\mathrm{R}$ & $8.1 \pm 1.2 \mathrm{C}$ & $8.7 \pm 1.1 \mathrm{C}$ & $10.0 \pm 1.1 \mathrm{cb}$ & $12.7 \pm 0.9$ a & $13.2 \pm 2.2 \mathrm{a}$ & $12.0 \pm 2.9 a b$ \\
\hline $\mathrm{Pa} / \mathrm{R}$ & $2.3 \pm 0.3 a$ & $2.5 \pm 0.6 a$ & $2.9 \pm 0.6 \mathrm{a}$ & $2.6 \pm 0.6 \mathrm{a}$ & $2.7 \pm 0.6 \mathrm{a}$ & $3.1 \pm 0.8 \mathrm{a}$ \\
\hline S & $0.4 \pm 0.1 \mathrm{a}$ & $0.30 \pm 0.1 \mathrm{a}$ & $0.33 \pm 0.0 \mathrm{a}$ & $0.37 \pm 0.1 \mathrm{a}$ & $0.41 \pm 0.1 \mathrm{a}$ & $0.39 \pm 0.1 \mathrm{a}$ \\
\hline AF & $529.4 \pm 61.0 c$ & $583.0 \pm 54.4 \mathrm{c}$ & $678.2 \pm 60.6 \mathrm{bc}$ & $781.5 \pm 55.5 b$ & $823.1 \pm 224.2 \mathrm{ab}$ & $965.6 \pm 87.7 \mathrm{a}$ \\
\hline AsT & $1.4 \pm 0.1 \mathrm{c}$ & $1.5 \pm 0.2 \mathrm{c}$ & $1.5 \pm 0.2 c$ & $1.7 \pm 0.2 b c$ & $2.6 \pm 0.6 \mathrm{a}$ & $2.0 \pm 0.3 b$ \\
\hline AV & $201.5 \pm 13.7 c$ & $185.9 \pm 24.4 \mathrm{c}$ & $218.6 \pm 9.0 \mathrm{c}$ & $274.9 \pm 25.1 b$ & $319.4 \pm 48.9 a$ & $263.5 \pm 14.5 b$ \\
\hline
\end{tabular}

Medias con la misma letra en las hileras no presentan diferencias estadísticamente significativas (Tukey, 0.05). La media se acompaña \pm la desviación estándar. Var: variable, MST: materia seca total (g), PA: MS aérea (g), R: MS en raíz (g), Pa/R: relación parte aérea/raíz, S: suculencia (cm³ $\mathrm{cm}^{-2}$ ), AF: área foliar $\left(\mathrm{cm}^{2}\right)$, AsT: área de la sección transversal de la parte basal de la hoja $\left(\mathrm{cm}^{2}\right)$, AV: haces vasculares en la sección transversal de la hoja. 
planta, principalmente nitrógeno, es un factor limitante del tamaño de su área foliar, contenido de clorofila foliar y fotosíntesis neta, que están directamente asociados con la magnitud de crecimiento y acumulación de materia seca (Boussadia et al., 2010).

\section{CONCLUSIONES}

Las plantas en vivero fertirrigadas durante $240 \mathrm{~d}$ con cantidades diferentes de nutrimentos generaron crecimiento diferenciado en área foliar, área de la sección transversal de la hoja y número de haces vasculares, materia seca acumulada en la parte aérea, en la raíz y total. La magnitud del crecimiento fue en relación positiva con la dosis de fertirrigación, alcanzando mayor tamaño aquellas plantas que recibieron solución nutritiva del 80 al $100 \%$ de la formulación de Steiner.

\section{BIBLIOGRAFÍA}

Angeles-Espino A., A. J. Valencia-Botín, G. Virgen-Calleros, C. Ramírez-Serrano, L. Paredes-Gutiérrez y S. Hurtado-De la Peña (2012) Micropropagación de agave (Agave tequilana Weber. var. Azul) a través de yemas axilares. Tropical and Subtropical Agroecosystems 15:693-698.

Barrera Z. V. A., T. Lawson, E. Olmos, N. Fernández-García and A. M. Borland (2014) Leaf anatomical traits which accommodate the facultative engagement of crassulacean acid metabolism in tropical trees of the genus Clusia. Journal of Experimental Botany 65:3513-3523. https://doi.org/10.1093/jxb/eru022.

Boussadia 0., K. Steppe, H. Zgallai, S. Ben El Hadj, M. Braham, R. Lemeur and M. C. Van Labeke (2010) Effects of nitrogen deficiency on leaf photosynthesis, carbohydrate status and biomass production in two olive cultivars 'Meski' and 'Koroneiki'. Scientia Horticulturae 123:336-342. https://doi.org/10.1016/j.scienta.2009.09.023

Enríquez-del Valle J. R., I. Cruz-Valdez and G. Carrillo-Castañeda (2012) Acclimatization of Agave angustifolia Haw. vitroplants in inert substrates and fertigated with different nutrimental dose. Acta Horticulturae 947:101-104.

Enríquez V. J. R., A. Estrada S., G. Rodríguez 0., V. A. Velasco V. y G. V. Campos A. (2013) Sustrato y dosis de fertirriego en la aclimatización de vitroplantas de Agave americana var. oaxacencis. Revista de la Facultad de Ciencias Agrarias. Universidad Nacional de Cuyo 45:341-348.

Enríquez V. J. R., S. E. Alcara V., G. Rodríguez O., M. E. Miguel L. y C. Manuel V. (2016) Fertirriego en vivero a plantas de Agave potatorum Zucc micropropagadas-aclimatizadas. Revista Mexicana de Cien- cias Agrícolas 7:1167-1177

Garriga C. M., G. González O., S. Alemán G., E. Abreu C., K. Quiroz B., P. D. S. Caligari and R. García-González (2010) Management of auxincytokinin interactions to improve micropropagation protocol of henequen (Agave fourcroydes Lem.). Chilean Journal of Agricultural Research 70:545-551, http://dx.doi.org/10.4067/ S0718-58392010000400003.

Mantovani A. (1999) A method to improve leaf succulence quantification. Brazilian Archives of Biology and Technology 42. http:// dx.doi.org/10.1590/S1516-89131999000100002

Martínez R. S., A. Trinidad S., C. Robles, A. Galvis S., T. M. Hernández M., J. A. Santizo R., G. Bautista S. y E. C. Pedro S. (2012) Crecimiento y sólidos solubles de Agave potatorum Zucc. inducidos por riego y fertilización. Revista Fitotecnia Mexicana 35:61-68

Martínez-Ramírez S., G. Bautista-Sánchez, E. C. Pedro-Santos y P. D. Guerrero-Cruz (2014) Crecimiento y contenido de clorofila del maguey mezcalero (Agave potatorum Zucc.) en policultivo con maíz y frijol. Revista Fitotecnia Mexicana 37:297-304.

Monja-Mio K. M., F. Barredo P., G. Herrera H., M. Esqueda V. and M. L. Robert (2015) Development of the stomatal complex and leaf surface of Agave angustifolia Haw. 'Bacanora' plantlets during the in vitro to ex vitro transition process. Scientia Horticulturae 189:3240. https://doi.org/10.1016/j.scienta.2015.03.032.

Pérez Molphe B. E., M. J. Esparza A. y M. E. Pérez R. (2012) Conservación in vitro de germoplasma de Agave spp. bajo condiciones de crecimiento retardado. Revista Fitotecnia Mexicana 35:279-287.

Pospišilová J., D. Haisel, H. Synková, J. Čatský, N. Wilhelmová, Š. Plzáková, D. Procházková and F. Šrámek (2000) Photosynthetic pigments and gas exchange during ex vitro acclimation of tobacco plants as affected by $\mathrm{CO}_{2}$ supply and abscisic acid. Plant Cell, Tissue and Organ Culture 61:125-133.

SAS Institute (2002) SAS User's Guide: Statistics. Version 9.0. SAS Institute. Cary, North Carolina, USA. 1032 p.

Salisbury F. B. and C. W. Ross (1992) Plant Physiology. 4th edition. Wadsworth Publishing Co. Belmont, CA, USA. $682 \mathrm{p}$

Santíz J. A., R. Rincón-Rosales y F. A. Gutiérrez-Miceli (2012) Propagación in vitro de Agave grijalvensis B. Ullrich, una especie endémica de Chiapas bajo protección especial. Gayana Botánica 69:2330.

SEMARNAT, Secretaría de Medio Ambiente y Recursos Naturales (2002) Norma Oficial Mexicana NOM-021-RECNAT-2000. Especificaciones de fertilidad, salinidad y clasificación de suelos. Estudios, muestreo y análisis. Diario Oficial de la Federación. Segunda Sección. 31 de diciembre de 2002. México, D.F. 73 p.

SEMARNAT, Secretaría de Medio Ambiente y Recursos Naturales (2010) Norma Oficial Mexicana NOM-059-SEMARNAT-2010. Protección ambiental. Especies nativas de México de flora y fauna silvestres. Categorías de riesgo y especificaciones para su inclusión exclusión o cambio. Lista de especies en riesgo. Diario Oficial de la Federación, Segunda Sección. 30 de diciembre de 2010. México, D.F. 78 p.

Steiner A. A. (1984) The universal nutrient solution. In: Proceedings of IWOSC 1984 6th International Congress on Soilless Culture. April 29-May 5, 1984. Wageningen, The Netherlands. pp:633650. 